Available online on 15.09 .2020 at http://jddtonline.info
ODDT * 5

Open $\odot$ Access

Research Article

\title{
Mucoadhesive microspheres based formulation development of ziprasidone hydrochloride for nasal delivery
}

\author{
Yogita Sahu1, Sourabh Jain*2, Karunakar Shukla² \\ ${ }^{1}$ Central India Institute of Pharmacy, Indore Bypass Rd, Manglaya Sadak, Indore, MP 453771, India \\ ${ }^{2}$ College of Pharmacy, Dr. A. P. J. Abdul Kalam University, Village, Post, Indore Bypass Rd, Arandia, Jhalariya, Madhya Pradesh 452016, India
}

\begin{abstract}
The purpose of research work was to develop and optimize mucoadhesive microspheres of Ziprasidone hydrochloride for nasal delivery with the aim to enhance the residence time and improve therapeutic efficacy. Mucoadhesive drug delivery systems are those that provide intimate contact of the drug with the mucosa for an extended period of time. In our present work, mucoadhesive chitosan microspheres were prepared by emulsification method using liquid paraffin as external phase. Ten different formulations were developed. Results show that as the concentration of polymer increases it affects the particle size, production yield, encapsulation efficiency, swelling index, in-vitro mucoadhesion and in-vitro drug release of mucoadhesive microspheres. The in vitro mucoadhesion of microspheres was investigated using freshly isolated goat nasal mucosa. The mucoadhesion for M0, M1, M2, and M9 was tested. The mucoadhesion property was satisfactory. The M2 exhibited lowest mucoadhesion of $68.9 \%$, and M0 displayed highest mucoadhesion of $87.5 \%$. The In Vitro release studies it revealed that $84.1 \%$ of drug release from formulation M1 at 7 hrs. The $50 \%$ of the drug was released from the formulation M2 and $70.67 \%$ from formulation M9.This formulations were further used for SEM for particles size analysis, mucoadhesion test and in-vitro drug release. The In-vitro \% drug release data suggest that the maximum and sustained drug release was obtained for formulation M1.The present study showed that Ziprasidone hydrochloride chitosan microspheres can deliver intanasally which can improve the therapeutic outcome for the Epileptic seizure.
\end{abstract}

Keywords: Ziprasidone hydrochloride, Mucoadhesive microspheres, Nasal drug delivery, Drug Entrapment efficiency.

Article Info: Received 14 July 2020; Review Completed 23 Aug 2020; Accepted 30 August 2020; Available online 15 September 2020

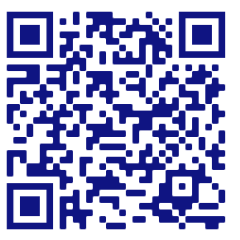

Cite this article as:

Sahu Y, Jain S, Shukla K, Mucoadhesive microspheres based formulation development of ziprasidone hydrochloride for nasal delivery, Journal of Drug Delivery and Therapeutics. 2020; 10(5):175-181

http://dx.doi.org/10.22270/jddt.v10i5.4309

Dr. Sourabh Jain, College of Pharmacy, Dr. A. P. J. Abdul Kalam University, Village, Post, Indore Bypass Rd, Arandia, Jhalariya, MP 452016

\section{INTRODUCTION}

Mucoadhesive drug delivery systems are those systems that utilize bioadhesion of certain polymers that become adhesive on hydration 1 . Thus, they can be used for targeting a drug to a particular region of the body for extended period of time 2 . The mucus membrane lines a number of regions of the body including the gastrointestinal tract (GIT), urogenital tract, airways, ear, nose, and eye. These are strong sites for attachment of any bioadhesive system, and hence the mucoadhesive drug delivery systems include 3 buccal, oral, vaginal, rectal, nasal, and ocular delivery systems. The nasal mucosa has far higher permeability than other mucosal surfaces including the various regions of the GIT, buccal, and vaginal cavities ${ }^{4}$. Recently, mucoadhesive dosage forms have received substantial attention to improve the bioavailability of drug by prolonging the residence time and controlling drug release characteristics 5 . Thus, mucoadhesion may lead to the solution of bioavailability problems resulting from a too-short stay of the pharmaceutical dosage form at the absorption site of the active ingredients. Faster absorption and easy administration through nasal cavity make the nasal drug delivery a promising route for administration of drugs. It appears to be an ideal alternative to the parenterals for administrating drugs intended for systemic effect in view of the rich vascularity of the nasal membrane and the ease of intranasal administration. The delivery of drug by this route has distinct advantages of protecting the drug from the hepatic first-pass elimination, gut wall metabolism, and destruction in GIT, and it also offers excellent controlled release of drug and better systemic therapeutic response. Moreover, there exists a rich vasculature and a highly permeable structure in the nasal membranes for absorption 6. Ziprasidone hydrochloride was the fifth a typical antipsychotic for the treatment of schizophrenia and the intramuscular injection form of ziprasidone is approved for acute agitation in schizophrenic patients 7. Chitosan is a natural polymer that has mucoadhesive properties because of its positive charges at neutral $\mathrm{pH}$, which enable an ionic interaction with the negative charges of sialic acid residues 
on the mucus 8,9 . This highly mucoadhesive characteristics of chitosan provide a longer contact period for drug transport through nasal mucosa and prevents the clearance of the formulation via mucociliarly clearance mechanism 10 Therefore, chitosan microspheres have been extensively evaluated as a drug delivery system ${ }^{11-14}$. The objective of the present study is to formulate and evaluate mucoadhesive microspheres of Ziprasidone hydrochloride that will increase residence time in the nasal cavity and at the same time increase the local of absorption of drug and reducing systemic side effects and also to develop unique controlled delivery system for patients suffering from allergy and rhinitis. The microspheres were prepared by emulsification cross linking method in different ratio by using mucoadhesive polymer, chitosan.

\section{MATERIAL AND METHODS}

\section{Material}

Ziprasidone hydrochloride was obtained as a gift sample from CADILA Pharmaceutical Pvt. Ltd., Ahmedabad, Gujarat. All the chemicals and ingredients were of analytical grade and supplied from authentic suppliers i.e. Chitosan (Chemika Biochemika Reagents, India), n-Hexane (Merck, Germany) Sodium Lauryl Sulphate (SLS), liquid paraffin (Kasliwal Bros, Indore, India), Gluteraldehyde (GA) (25\% Aqueous Solution) (Spectrochem Pvt. Ltd, Mumbai, India), (Loba Chemie Pvt Ltd Mumbai, India).

\section{Methods}

\section{Identification and characterization of API and polymer}

\section{Assay of ziprasidone hydrochloride by UV spectrophotometry}

Ziprasidone Hydrochloride assay was performed by using UV spectrophotometric method mentioned in some articles (UV spectrophotometer, Shimadzu, Japan) and observations were compared with standards. When examinations were performed sample was scanned in the range of $300-500 \mathrm{~nm}$ maximum absorbance was observed in the range of $509 \mathrm{~nm}$ to $521 \mathrm{~nm}$ with the solvent methanol as mention in the article. ${ }^{15-17}$

\section{FTIR spectroscopy}

Fourier transform Infrared absorption spectrophotometry (FT-IR)) was performed and spectrum was compared with reference spectrum of Ziprasidone Hydrochloride and chitosan.

\section{Melting point determination}

Melting points of Ziprasidone Hydrochloride and Chitosan were determined with the help of melting point apparatus and compared with standards.

\section{DSC analysis}

Differential scanning calorimetric (DSC) study was carried out to confirm the thermal behavior of Ziprasidone Hydrochloride and chitosan ${ }^{18}$.

\section{Preparation of blank chitosan microspheres by emulsification}

Chitosan microspheres were prepared by simple w/o emulsification-cross linking process 19,20 by using liquid paraffin (heavy and light, 1:1). The hardened microspheres were separated by filtration and washing of microspheres were done for several times with hexane to remove oil. Finally, microspheres were washed with distilled water to remove unreacted GA. Solution was added slowly to liquid paraffin (heavy and light, 1:1) containing $0.2 \%$ (w/v) of SLS as stabilizing agent under constant stirring at the speed of $2000 \mathrm{rpm}$ for $15 \mathrm{~min}$ using a high speed mechanical stirrer. The microspheres were air dried for $24 \mathrm{hrs}$ and then stored in vacuum desiccator until further use.

\section{Preperation of ziprasidone hydrochloride loaded chitosan microspheres}

Ziprasidone Hydrochloride loaded chitosan microspheres were prepared by simple w/o emulsification-cross linking process using liquid paraffin (heavy and light, 1:1) as external phase. At first, Ziprasidone Hydrochloride was dissolved in $2 \mathrm{ml}$ of acetone and sonicated till a clear solution is obtained. Chitosan was dissolved in $2 \%$ aqueous acetic acid solution by continuously stirring and then solution of chitosan was added in the drug solution with continuous stirring at constant speed until a homogeneous solution was obtained. This solution was added slowly to liquid paraffin (heavy and light, 1:1) containing $0.2 \%(\mathrm{w} / \mathrm{v})$ of SLS as stabilizing agent under constant stirring at a speed $1800 \mathrm{rpm}$ for 15 min using a high speed mechanical stirrer. To this w/o emulsion, GA was added slowly and stirring was continued for 2 hrs. The hardened microspheres were separated by vacuum filtration and washed several times with hexane to remove oily part. Finally, microspheres were washed with distilled water to remove unreacted GA. The microspheres were air dried for $24 \mathrm{~h}$ and then stored in desiccator until further use. To evaluate the effect of different formulation and process variables on particle size, encapsulation efficiency, drug loading etc., various batch of formulations were prepared by varying one parameter and keeping the others constant as given in Table 1.

Table 1: composition of Ziprasidone hydrochloride mucoadhesive microspheres

\begin{tabular}{|c|c|c|c|c|c|c|}
\hline F. No. & $\begin{array}{c}\text { Drug: Polymer } \\
\text { (Ratio) }\end{array}$ & $\begin{array}{c}\text { Aqueous : Oil } \\
\text { (Ratio) }\end{array}$ & $\begin{array}{c}\text { GA } \\
\text { (ml) }\end{array}$ & $\begin{array}{c}\text { SLS } \\
\text { (\%) }\end{array}$ & $\begin{array}{c}\text { Stirring Rate } \\
\text { (rpm) }\end{array}$ & $\begin{array}{c}\text { Cross Linking Time } \\
\text { (Hrs) }\end{array}$ \\
\hline M0 & $0: 10$ & $10: 100$ & 2 & 0.2 & 1800 & 2 \\
\hline M1 & $1: 9$ & $10: 100$ & 2 & 0.2 & 1800 & 2 \\
\hline M2 & $2: 8$ & $10: 100$ & 2 & 0.2 & 1800 & 2 \\
\hline M3 & $3: 7$ & $10: 100$ & 2 & 0.2 & 1800 & 2 \\
\hline M4 & $1: 9$ & $20: 100$ & 2 & 0.2 & 1800 & 2 \\
\hline M5 & $1: 9$ & $10: 100$ & 2 & 0.2 & 1800 & 2 \\
\hline M6 & $1: 9$ & $10: 100$ & 2 & 0.2 & 1200 & 2 \\
\hline M7 & $1: 9$ & $10: 100$ & 2 & 0.2 & 1500 & \\
\hline M8 & $1: 9$ & $10: 100$ & 2 & 0.1 & 1800 & 2 \\
\hline M9 & $1: 9$ & $10: 100$ & 2 & 0.2 & 1800 & 2 \\
\hline
\end{tabular}




\section{Characterization of microspheres}

\section{Production yield (\%)}

Production yield of microspheres of various batches were calculated using the weight of final product after drying with respect to the initial total weight of the drug and polymer used for preparation of microspheres and \% production yields were calculated by formula 21 .

\section{Degree of swelling}

The swell ability of microspheres in physiological media was determined by swelling them in the phosphate buffer solution of $\mathrm{pH}$ 6.4. Accurately weighed amount of microspheres (10 mg) was immersed in little excess of PBS $\mathrm{pH} 6.4$ for $24 \mathrm{hrs}$ followed by washing. The degree of swelling was calculated using following method: A known weight $(50 \mathrm{mg})$ of microspheres were placed in a glass vial containing $10 \mathrm{~mL}$ phosphate buffer of $\mathrm{pH} 6.8$ and maintained at $37 \pm 0.5^{\circ} \mathrm{C}$ with gentle shaking for $24 \mathrm{~h}$. After that, microspheres were removed, blotted with filter paper and subsequently, the weight of the swollen microspheres (Wsw) was measured 22 .

The swelling ratio (SR) was calculated according to the following equation

$$
\propto=\frac{\mathbf{W}_{\mathbf{S}}-\mathbf{W}_{\mathbf{0}}}{\mathbf{W}_{\mathbf{0}}}
$$

Where $\propto$ = degree of swelling

$\mathrm{W}_{0}=$ weight of microspheres before swelling and

$\mathrm{W}_{\mathrm{s}}=$ weight of microspheres after swelling.

\section{In vitro mucoadhesion studies 23}

Mucoadhesion of microspheres was assessed by using the method reported with little modification. The in vitro mucoadhesion of microspheres was investigated by using freshly isolated goat nasal mucosa. In brief, nasal mucosal tissues were carefully removed from the nasal cavity of goat, which was obtained from the local slaughter house within 60 minutes of slaughtering the animal. The tissues were cut into the size of $1 \times 1 \mathrm{~cm}$ and were mounted onto the glass slide and accurately weighed microspheres (10 mg) were sprinkled on the mucosa. This glass slide was kept in desiccator for $15 \mathrm{~min}$ to allow the polymer to interact with the membrane and finally placed in the cell that was attached to the outer assembly at an angle of $45^{\circ}$. Phosphate buffer solution $\mathrm{pH} 6.4$, previously warmed to $37 \pm 5^{\circ} \mathrm{C}$ was circulated all over the microspheres and membrane at the rate of $1 \mathrm{ml} / \mathrm{min}$. Washings were collected at different time intervals and microspheres were collected by centrifugation followed by drying at $50^{\circ} \mathrm{C}$. The weight of washed out microspheres was determined and percent mucoadhesion was calculated by standard formula.

\section{External morphological evaluation}

\section{Optical microscopy}

All batches of microspheres were studied for shape and size by optical microscopy (Olympus Microscope, Olympus Optical Co. Ltd., Japan). The samples were studied in the form of dispersion in paraffin oil.
In vitro drug release / diffusion kinetics of polymeric microspheres 24

The drug release experiments employed Franz diffusion cells, since this model would allow the microspheres to hydrate slowly in a humid environment, conditions designed to be similar to those encountered in the nasal cavity. Goat nasal mucosa was obtained from local slaughter house. It was kept in phosphate buffer ( $\mathrm{pH}$ 6.8) and then cartilage was removed carefully. Tissue was stabilized and mounted in Franz diffusion cell containing phosphate buffer of $\mathrm{pH} 6.8$ in acceptor compartment. Weighed quantity of microspheres (moistened with phosphate buffer), were applied evenly across a nasal mucosal membrane of goat mounted in a Franz diffusion cell. The Acceptor compartment was filled with a known amount of buffer solution ( $\mathrm{pH}$ 6.8) as a receptor medium, which was stirred with a magnetic stirring bar. The assembled cells were placed in a water bath (37 \pm $0.2^{\circ} \mathrm{C}$ ) in a $100 \%$ humidity. At set time intervals, $0.5 \mathrm{ml}$ was removed via the side arm and assayed for Ziprasidone Hydrochloride at $521 \mathrm{~nm}$ by UV-visible spectrophotometer. The removed samples were replaced with an equal quantity of pre-warmed fresh buffer. Each of the formulation was analyzed by this method to study the effect of different process variables on the microspheres.

\section{RESULT AND DISCUSSION}

\section{Identification and characterization of API and polymer}

\section{Assay of ziprasidone hydrochloride by UV} spectrophotometry

The Ziprasidone Hydrochloride was identified by UV spectroscopy method. The Ziprasidone Hydrochloride exhibited maximum absorption at $521 \mathrm{~nm}$. After scanning the $\lambda_{\max }$ of the Ziprasidone Hydrochloride with methanol it matches with that of the standard $\lambda_{\max }$ given in the articles $(521 \mathrm{~nm})$. This wavelength was considered as $\lambda_{\max }$ for samples and all the observations by UV spectrophotometer to calculate the amount of drug was taken at this wavelength. The Calibration curve of Ziprasidone Hydrochloride is shown on Fig.1.

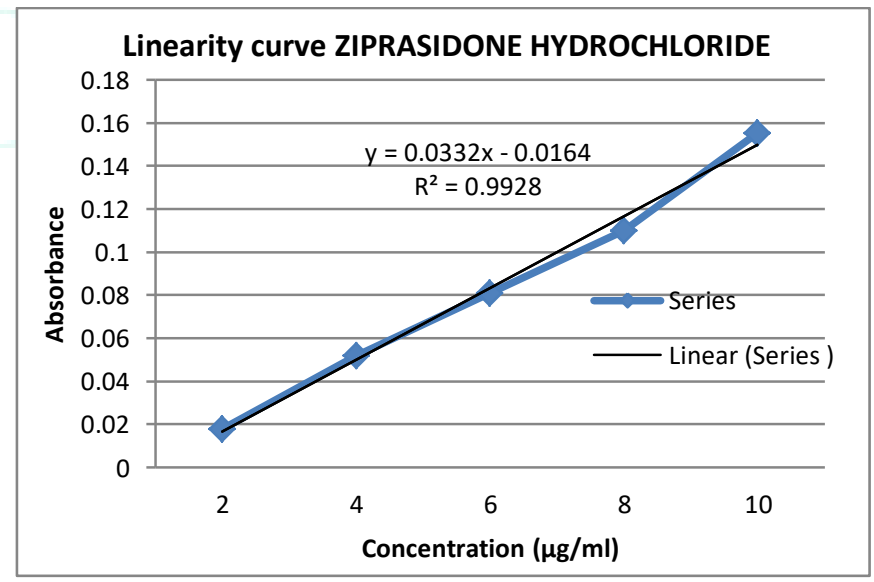

Figure 1: Calibration curve of ziprasidone hydrochloride drug in methanol

\section{FTIR spectroscopy}

The spectra of FTIR (Fig.2) and its description and interpretation in the below tables indicate that the sample used was Ziprasidone hydrochloride. 

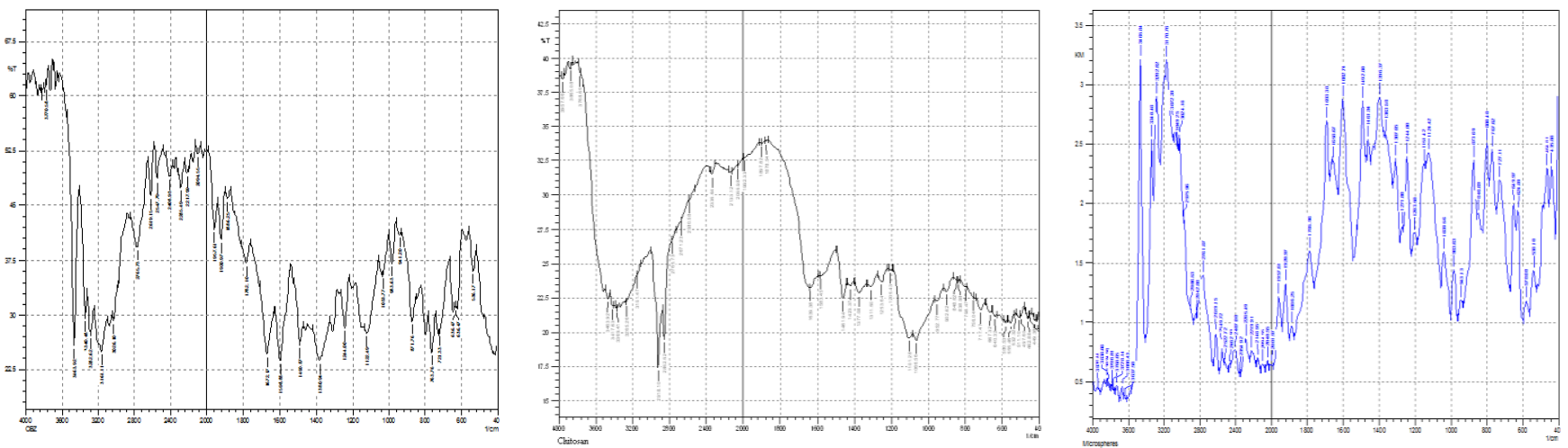

Figure 2: FT-IR Spectra of ziprasidone hydrochloride, chitosan and formulation

\section{DSC analysis}

From Figure 3 it has been observed that the drug endothermic peak disappeared. It has been reportedthat when the drug does not show its endothermic peak in the microsphere, it is said to be in the amorphous state or disordered crystalline phaseas a molecular dispersion in polymeric matrix. Hence, it could be concluded that the drug was present in the amorphous or solid phase and may have been homogeneously dispersed in the microsphere.
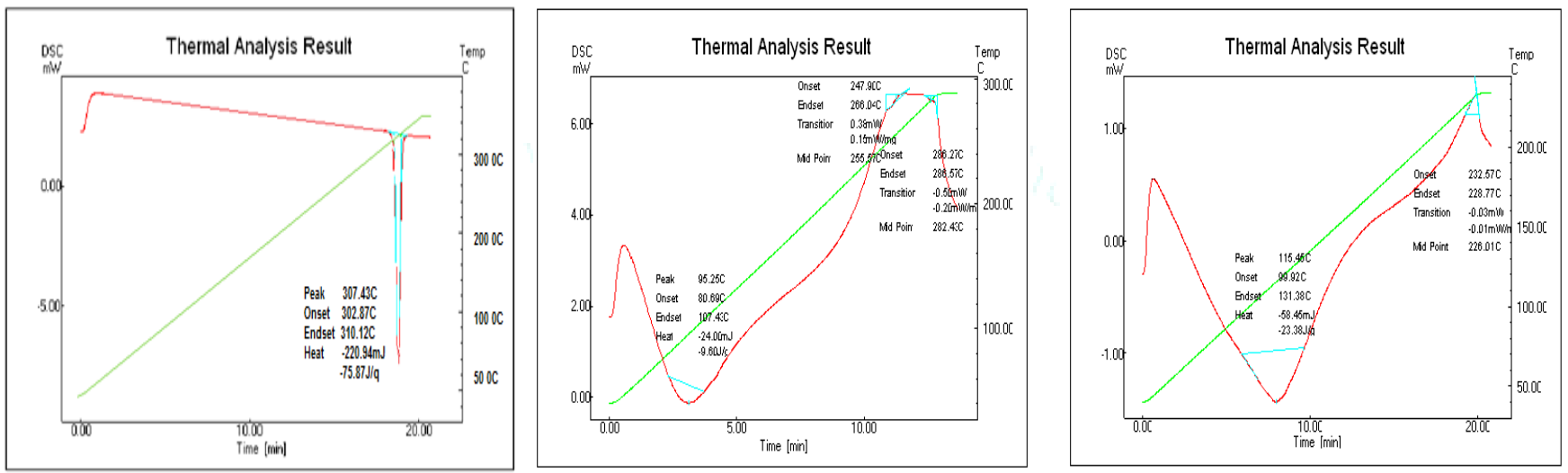

Figure 3: DSC thermogram of ziprasidone hydrochloride, chitosan and formulation

\section{Characterization and evaluation of microspheres}

Ten formulation of ziprasidone hydrochloride microspheres were prepared to optimize the formula. The effect of drugpolymer ratio, aqueous-oil ratio, quantity of dispersing agent, GA and stirring rate of stirrer on particle geometry of microsphere has been studied.

\section{Optical microscopy}

Microspheres were characterized in terms of sphericity and clumping of microspheres by optical microscopy. Optical microscopy of drug loaded microspheres depicted irregular geometry in some batches due to which those batches fails to achieve better encapsulation. Here we are discussing geometry of all the batches and observed almost spherical geometry with few aggregates in some formulations. Formulations M0 and M1 shows uniform spherical shapes with no aggregation of spherical. On the other way formulation M2 shows non-uniform size and shapes of particles with no aggregation. Formulation M3 shows irregular and degraded particles with no aggregation. Formulation M4 represents aggregated and clumsy particles and on the other hand formulation M5 shows dense aggregation of particles. Formulations M6 and M7 shows clumping of particles with irregular geometry. Formulation M8 shows lumps formation and at last formulation M9 shows spherical shaped microspheres with no aggregation. The findings of optical microscopy suggest that the particle of microspheres of M0, M1 and M9 were satisfactory Fig. 4.
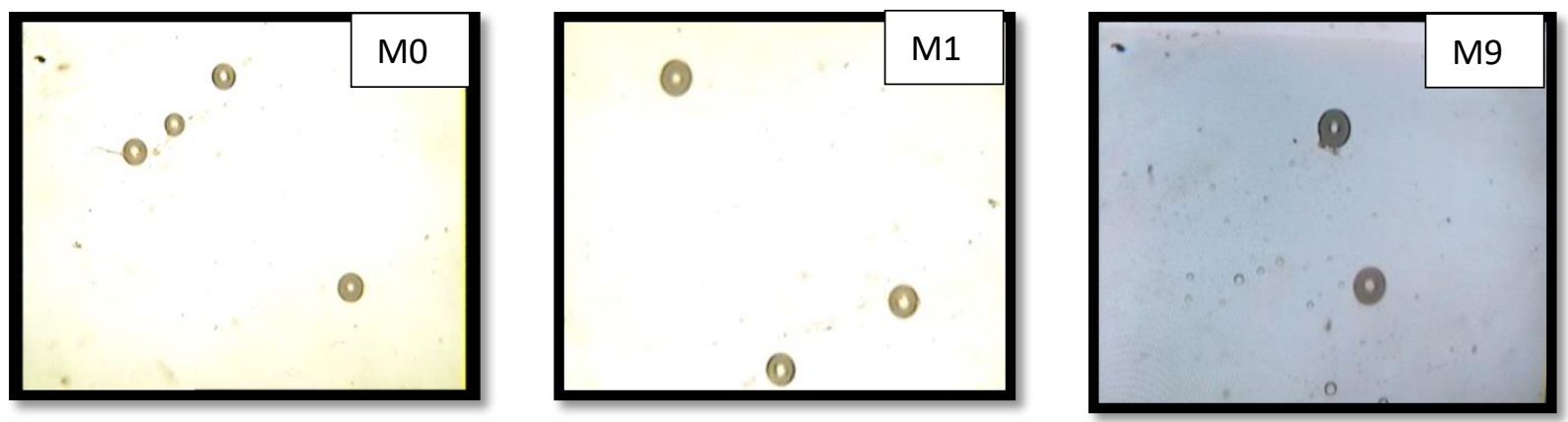

Figure 4: Microscopical images of satisfactory batches (M0, M1, M9) 


\section{Production yield (\%)}

It was recorded that as concentration of polymer increases production yield decreases, since increase in polymeric concentration make solution more viscous this was difficult to pour and get sticked on the wall of the beaker. The \% production yield of microspheres was found to be maximum for M3 (74.59\%) having lowest amount of polymer. Lowest $\%$ yield was of formulation M4 (51.56\%). Stirring rate also affects production yield Table 2 .

Table 2: Obtained production yield of all the formulations

\begin{tabular}{|c|c|c|c|}
\hline Formulation No. & Practical mass (mg) & Theoretical mass (mg) & Production yield (\%) \\
\hline M0 & 60.24 & 100 & 60.24 \\
\hline M1 & 63.12 & 100 & 63.12 \\
\hline M2 & 71.89 & 100 & 71.89 \\
\hline M3 & 74.11 & 100 & 74.11 \\
\hline M4 & 51.56 & 100 & 51.56 \\
\hline M5 & 54.03 & 100 & 54.03 \\
\hline M6 & 58.43 & 100 & 58.43 \\
\hline M7 & 50.01 & 100 & 50.01 \\
\hline M8 & 61.78 & 100 & 61.78 \\
\hline M9 & 64.21 & 100 & 64.21 \\
\hline
\end{tabular}

\section{Entrapment efficiency (\%)}

The entrapment efficiencies of drug were recorded in the range of $48.05 \%$ to $79 \%$ for M1 to M9. The M8 revealed lowest entrapment efficiency of drug i.e. $48.09 \%$, while M1 displayed $79 \%$ highest drug entrapment efficiency when compared to other formulations. From results it has been observed that on increasing the polymer ratio the drug entrapment efficiency increases. This might be due to higher concentration of polymer make solution highly viscous which increases the drug entrapment. Meanwhile increasing the stirring rate also enhances the drug entrapment in microspheres because increasing the stirring rate decreases the particle size of microsphere and surface area of microsphere enhanced, this intensifies the drug permeability inside the microspheres. It is another reason that can which increases the drug entrapment efficiency in microspheres Table 3.
Table 3: Entrapment efficiency of microspheres of drug load formulations

\begin{tabular}{|c|c|}
\hline Formulations & Entrapment Efficiency (\%) \\
\hline M1 & 79.54 \\
\hline M2 & 62.87 \\
\hline M3 & 56.18 \\
\hline M4 & 71.23 \\
\hline M5 & 71.65 \\
\hline M6 & 50.34 \\
\hline M7 & 52.76 \\
\hline M8 & 48.09 \\
\hline M9 & 70.65 \\
\hline
\end{tabular}

\section{Degree of swelling index}

The degree of swelling was ranged from 0.36 to $1.66 \%$ for formulations M0 to M9. The lowest degree of swelling was observed in M3 $(0.36 \%)$ and the highest degree of swelling was noticed in M0 (1.66\%) Table 4.

Table 4: Degree of swelling of microspheres

\begin{tabular}{|c|c|c|c|}
\hline Formulations & $\begin{array}{c}\text { Weight of microspheres before } \\
\text { swelling }\end{array}$ & $\begin{array}{c}\text { Weight of microspheres after } \\
\text { swelling }\end{array}$ & Degree of Swelling (\%) \\
\hline M0 & 60.24 & 61.65 & 1.66 \\
\hline M1 & 63.12 & 63.96 & 1.33 \\
\hline M2 & 71.89 & 72.19 & 0.41 \\
\hline M3 & 74.11 & 75.88 & 0.36 \\
\hline M4 & 51.56 & 51.86 & 0.58 \\
\hline M5 & 54.03 & 54.24 & 0.38 \\
\hline M6 & 58.43 & 58.98 & 0.941 \\
\hline M7 & 60.01 & 60.59 & 0.966 \\
\hline M8 & 51.78 & 51.95 & 0.57 \\
\hline M9 & 64.21 & 65.01 & 1.24 \\
\hline
\end{tabular}


The results of the studies revealed that, swelling ratio of the microspheres increased with increasing the ratio of the polymer which could be due to more amount of polymer present in the microspheres that may be responsible for higher swelling. Swelling ratios were also increased with reduced particle size of the microspheres. This could be probably due to the reason that when the particle size is reduced, more surface area will be exposed to the swelling medium and consequently leads to the higher swelling of the microspheres. Swelling ratios of the microspheres reduced when the extent of cross-linking was increased. This could be due to fact that, higher concentration of gluteraldehyde which dense the cross-linking of polymeric chains thereby limiting the penetration of the medium in to the polymeric matrix thereby reducing the swelling ability of the microspheres. On the bases of above evalutions only four formulations M0, M1, M2 and M9 were selected for further tests.

\section{Mucoadhesion test or In-vitro washes off test}

On the basis of above evalution only four formulations were selected for further tests. The mucoadhesion for M0, M1, M2, and M9 was tested. The Mucoadhesion property was satisfactory. The M2 exhibited lowest mucoadhesion of $68.9 \%$, and M0 displayed highest mucoadhesion of $87.5 \%$. The results showed that M0, M1, M9 had satisfactory mucoadhesive strength and could adequately adhere to nasal mucosa. Tensile studies performed with ziprasidone hydrochloride loaded microsphere showed that drug/polymer ratio significantly influenced the mucoadhesive properties of the microspheres. Hence high concentration of polymer imparts larger penetration with maximum adhesion Thus, formulation M0, M1 and M9 satisfy the mucoadhesion Table 5.

Table 5: Mucoadhesion of microspheres

\begin{tabular}{|c|c|c|c|}
\hline Formulations & $\begin{array}{c}\text { Weight of microspheres } \\
\text { applied (mg) }\end{array}$ & $\begin{array}{c}\text { Weight of microspheres } \\
\text { leached out (mg) }\end{array}$ & Mucoadhesion (\%) \\
\hline M0 & 10 & 1.25 & 84.5 \\
\hline M1 & 10 & 1.52 & 68.9 \\
\hline M2 & 10 & 3.11 & 74.1 \\
\hline M9 & 10 & 2.59 & \\
\hline
\end{tabular}

From the results of mean particle size, production yield, entrapment efficiency, drug loading capacity, degree of swelling and mucoadhesion indicates that 3 formulations yield best microspheres over, all the parameters. Hence below mentioned 3 formulations were proceeds for further microscopic studies

\section{Morphological characteristics of microsphere by SEM}

The surface morphology of the drug loaded microsphere was investigated by scanning electron microscopy. Studies using SEM provided a better understanding of the morphological characteristics of the microspheres Fig. 5.
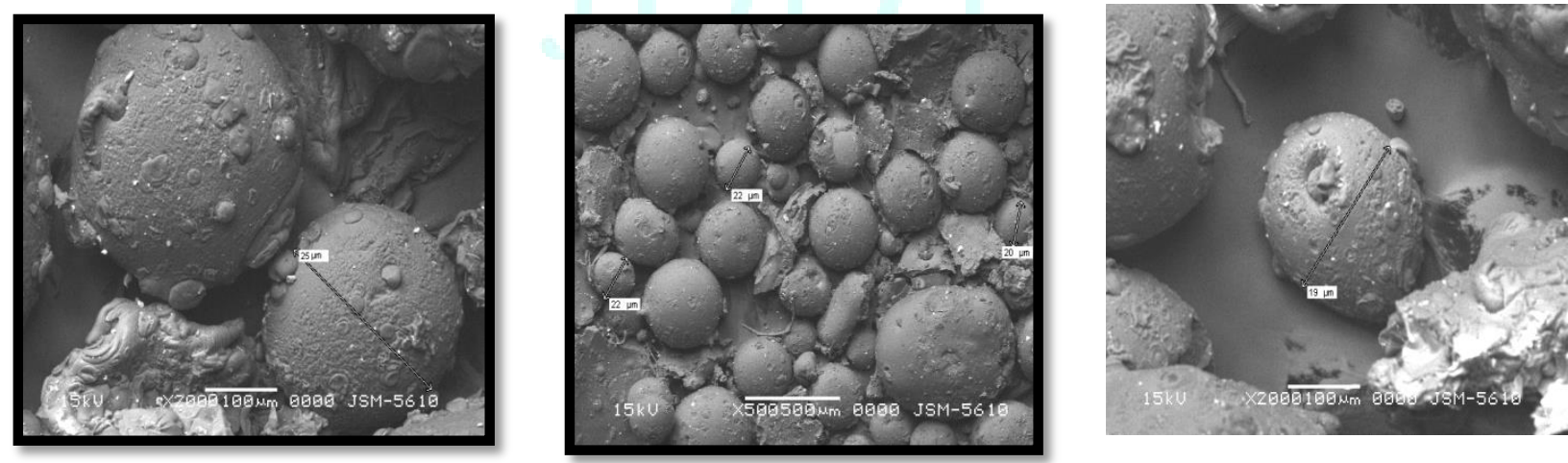

Figure 5: Microspheres of formulation M1, M2 and M9 respectively.

\section{In vitro drug release studies}

From the in vitro release studies it revealed that $84.1 \%$ of drug release from formulation M1 at 7 hrs. The $\mathbf{5 0 \%}$ of the drug was released from the formulation M2 and $70.67 \%$ from formulation M9. From above finding it has been noticed that on increasing the concentration of chitosan it decreased the drug release from microspheres. The high stirring rate, high concentration of chitosan and GA make the microsphere stiff. This hardness of microsphere decreases the rate of drug release from microspheres. Thus formulation M1 exhibited the sustained release of drug from microspheres in comparison to others Fig. 6. 

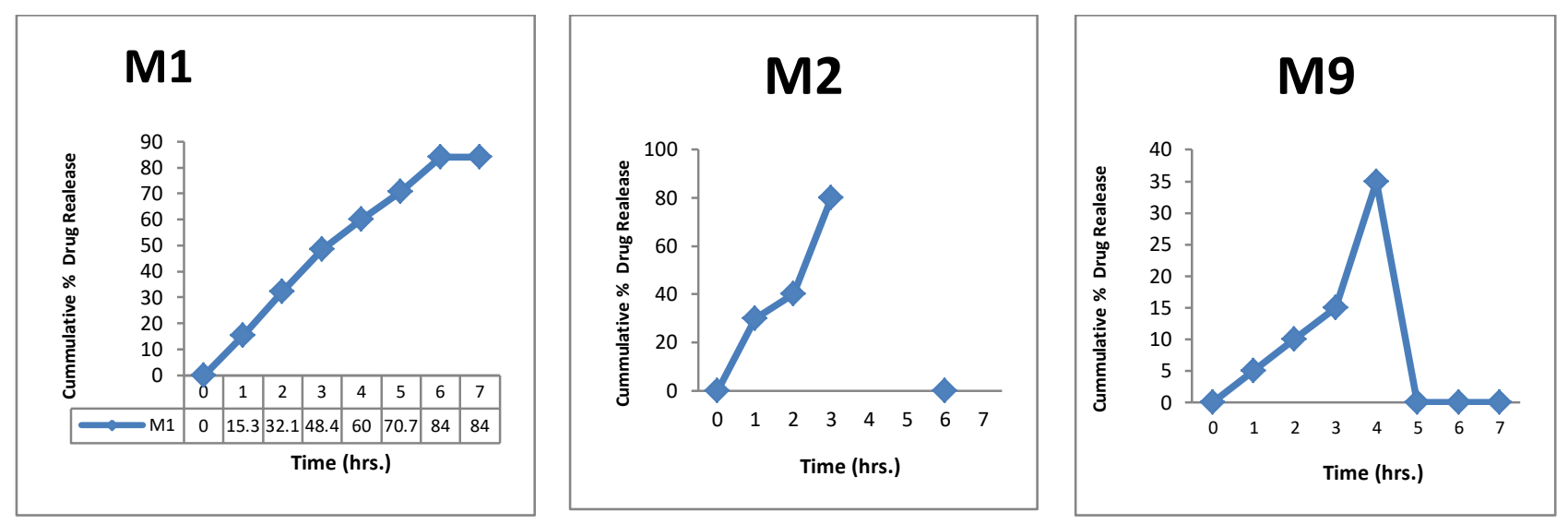

Figure 6: Cumulative drug release of formulation M1, M2 and M9

\section{CONCLUSION}

In the present study, an attempt was made to develop mucoadhesive nasal microspheres for systemic delivery of Ziprasidone Hydrochloride. The method of preparation used is Emulsification-cross linking process due to the nature of drug. Different formulation batches were evaluated for various parameters like production yield, entrapment efficiency, swelling Index and particle geometry determination were found to get a better result for formulation M1, M2 and M9.This formulations were further used for SEM for particles size analysis, mucoadhesion test and in-vitro drug release. The particle size of formulations was found to be satisfactory. The In-vitro mucoadhesive study demonstrated that increase in polymer concentration, increases adherence of microspheres to mucus to a greater extent. The In-vitro \% drug release data suggest that the maximum and sustained drug release was obtained for formulation M1. In conclusion, the present study showed that Ziprasidone chitosan microspheres can deliver intanasally which can improve the therapeutic outcome for the Epileptic seizure.

\section{REFERENCES}

1. Nagai $\mathrm{T}$, Machida $\mathrm{Y}$ Advances in drug delivery: mucosal adhesive dosage forms. Pharm Int, 1985; 6:196-200.

2. Kamath KR, Park K, Sugbrick J, Boylen JC. Encyclopedia of Pharmaceutical Technology. New York: Marcel Dekker, 1994.

3. Jimen J, Castllanos MR, Zia H, Rhodes CT. Mucoadhesive drug delivery systems. Drug Dev Ind Pharm 1994; 19:143-154.

4. Illum L. Chitosan and its use as a pharmaceutical excipients. Pharm Res 1998; 15:1326-1331.

5. Takeuchi H, Yamamoto H, Niwa T, Hino T, Kawashima Y. Mucoadhesion of polymer coated liposomes to rat intestine in vitro. Chem Pharm Bull 1994; 42:1954-1960.

6. Chien YW, Su KSE, Chang SF. Nasal Systemic Drug Delivery. New York: Marcel Dekker. 1989.

7. El-Say KM, ElHelw AM, Ahmed OAA, Hosny KM, et al. Statistical optimization of controlled release microspheres containing cetirizine hydrochloride as a model for water soluble drugs. Informa Healthcare, 2014; 1-9.

8. Lehr CM, Bouwstra JA, Schacht EH, Junginger HE. In vitro evaluation of mucoadhesive properties of chitosan and some other natural polymers. Int J Pharm 1992; 78:43-8.

9. Singla AK, Chawla M. Chitosan, Some pharmaceutical and biological aspects-an update. I Pharm Pharmacol 2001; 53:1047-67.
10. Soane RJ, Frier M, Perkins AC, Jones NS, et al. Evaluation of the clearance characteristics of bioadhesive systems in humans. Int J Pharm 1999; 178:55-65.

11. Artursson P, Lindmark T, Davis SS, Illum L. Effect of chitosan on the permeability of monolayers of intestinal epithelial cells (Caco-2). Pharm Res 1994; 11:1358-61.

12. Sinha VR, Singla AK, Wadhawan S, Kaushik R, et al. Chitosan microspheres as a potential carrier for drugs. Int J Pharm 2004; 274:1-33.

13. Hafner A, Filipovic-Grcic J, Voinovich D, Jalsenjak I. Development and in vitro characterization of chitosanbased microspheres for nasal delivery of promethazine. Drug Dev Ind Pharm 2007; 33:427-36.

14. Asane GS, Nirmal SA, Rasal KB, Naik AA, et al. Polymers for mucoadhesive drug delivery system: A current status. Drug Dev Ind Pharm. 2008; 34:1246-66

15. Borse MP, Mulgund SV. UV Spectrophotometric estimation of ziprasidonein bulk and tablet dosage form. Der Pharm Lett, 2015; 7(5):272-275.

16. Varma MM, Begum R. Formulation, physicochemical evaluation, and dissolution studies of ziprasidonesolid dispersions. Int J Pharm Sci Nanotec, 2012; 5(3):56-59.

17. Kumar RT, Umamaheswari S. FTIR, FTR and UV-Vis analysis of carbamazepine. Res J Pharm Biol Chem, 2011; 2(4):78-84.

18. Pajander J, Rensonnet A, Øvergård M, Olsen MR, et al. Solid form change of Ziprasidoneduring hot melt processing. Thermochimica Acta 1995; 248:259-269.

19. Mitra A, Dey B. Chitosan microspheres in novel drug delivery systems. Indian J Pharm Sci. 2011; 73(4):355-366.

20. El-Hameed MDA, Kellaway IW. Preparation and in vitro characterisation of mucoadhesive polymeric microspheres as intra-nasal delivery systems. Eur J Pharm Biopharm 1997; 44:53-60.

21. Lamprecht A, Torres HR, Schäfer U, Lehr CM. Biodegradable microparticles as a two-drug controlled release formulation: a potential treatment of inflammatory bowel disease. J Contr Rel. 2000; 69:445-454.

22. Doddayya H, Srishailgouda SP, Reddy BT, Kumar P, et al. formulation and evaluation of brain-targeted nasal selegiline hydrochloride microspheres. Int J Pharm Biomed Res 2014; 20:123-156.

23. Gavini E, Hegge AB, Rassu G, Sanna V, et al. nasal administration of ziprasidoneusing chitosan microspheres: in vitro/in vivo studies. Int J Pharm.2006; 307:9-15.

24. Charulatha R, Rajan RK. Design and evaluation of Ziprasidone controlled release drug delivery system. Int J Pharm Tech Research, 2012; 2:23-45. 